\title{
Modulation of Cardiocyte Functional Activity by Antibodies against Trypanosoma cruzi Ribosomal P2 Protein C Terminus
}

\author{
P. SEPULVEDA, ${ }^{1} \dagger$ P. LIEGEARD,${ }^{1}$ G. WALLUKAT, ${ }^{2}$ M. J. LEVIN, ${ }^{3}$ AND M. HONTEBEYRIE ${ }^{1 *}$ \\ Laboratoire d'Immunopathogenèse, Département d'Immunologie, Institut Pasteur, 75724 Paris Cedex 15, France ${ }^{1}$; \\ Department of Cardiology, Max Delbrück Center for Molecular Medicine, Berlin 13125, Germany²; \\ and INGEBI, 1428 Buenos Aires, Argentina ${ }^{3}$
}

Received 14 February 2000/Returned for modification 5 April 2000/Accepted 23 June 2000

\begin{abstract}
Antibodies against the Trypanosoma cruzi ribosomal $\mathrm{P} 2 \beta$ protein (TcP2 $\beta$ ) have been associated with the chronic cardiac pathology of Chagas' disease in humans. Using synthetic peptides spanning the entire TcP2 $\beta$ molecule, we investigated their epitope recognition by antibodies from mice chronically infected with $T$. cruzi and from mice immunized with two recombinant TcP2 $\beta$ s. We found clear differences in epitope recognition between antibodies from $T$. cruzi-infected mice and mice immunized with two different recombinant TcP2 $\beta$ s associated with different schedules of immunization. Major epitopes recognized by antibodies from mice immunized with recombinant glutathione $S$-transferase (GST) or histidine (Hist) fusion TcP2 $\beta$ (GST-TcP2 $\beta$ or HistTcP2 $\beta$ ) are located in the central and hinge regions of the molecule. Nevertheless, mice immunized with Hist-TcP2 $\beta$ were also able to elicit antibodies against the TcP2 $\beta \mathrm{C}$ terminus, a region which is highly conserved in both $T$. cruzi and mammal ribosomal $P$ proteins. Strikingly, antibodies from infected animals recognized only the TcP2 $\beta$ C terminus. By using these antisera with distinct profiles of epitope recognition, it could be shown that only $C$ terminus-specific antibodies were able to increase the beating frequency of cardiomyocytes from neonatal rats in vitro by selective stimulation of the $\beta 1$-adrenergic receptor. Thus, antibodies against the TcP2 $\beta \mathrm{C}$ terminus elicited in the absence of infection are able to modulate a functional activity of host cells through a molecular mimicry mechanism.
\end{abstract}

Chagas' disease, caused by the protozoan Trypanosoma cruzi, is a tropical disease affecting most Latin American countries. In humans, the infection is usually detected during the chronic phase, either through routine serology in blood bank screenings or in patients in different stages of disease. Chagas' disease develops first as an acute phase characterized by patent parasitemia and general acute clinical signs of various magnitudes. A chronic phase follows spontaneously about 2 months later, when the parasitemia declines significantly and becomes subclinical. The complex pathology at chronicity also varies considerably, ranging from light cardiac symptoms to intense chronic cardiomyopathy leading to heart failure and death (32). In some areas, patients may also develop clinical pathology in the digestive tract of variable intensity with or without heart involvement (1).

The mechanisms responsible for the cardiomyopathy are not clearly understood, but the occurrence of chronic myocardial injury in the near absence of parasites suggests an autoimmune phenomenon (32). Nevertheless, the hypothesis of an autoimmune disorder in Chagas' disease remains controversial (16). Thus, cardiomyopathy might result from a parasite-induced polyclonal activation of the immune system leading to a breakdown of tolerance for self-antigens (34). Another possibility involves a $T$. cruzi-induced cross-reactive immune response to self-antigens through a molecular mimicry-dependent mechanism (6). Indeed, immune responses developed against selfantigens via molecular mimicry-dependent mechanisms have been shown to play a role in autoimmune phenomena associated with infectious diseases $(6,29)$. Several $T$. cruzi antigens

\footnotetext{
* Corresponding author. Mailing address: Département d'Immunologie, Institut Pasteur, 28, rue Dr. Roux, 75724 Paris Cedex 15, France. Phone: 331406135 17. Fax: 331406134 40. E-mail: mhj@pasteur .fr.

$\dagger$ Present address: C/Olivereta 40, 46018 Valencia, Spain.
}

have been reported to present epitopes similar to mammalian antigens, including the family of trypomastigote-specific Fl-160 antigens $(39,40)$, the microtubule associated-protein $(15)$, the cardiac myosin antigen (B13) $(14,38)$, and members of the acidic ribosomal $\mathrm{P}$ protein family $(24,31,33)$. Among the latter, the $T$. cruzi ribosomal $\mathrm{P} 1$ and $\mathrm{P} 2$ antigenic determinants are highly homologous at the $\mathrm{C}$ terminus with their human or mouse counterparts.

Patients with Chagas' heart disease develop antibodies against ribosomal $\mathrm{P} 1$ and $\mathrm{P} 2$ proteins $(\mathrm{TcP} 2 \beta)$ directed mainly against the $\mathrm{C}$ termini of these molecules. Moreover, the $\mathrm{C}$ terminus ribosomal P1-P2 peptide (R-13: EEDDDMGFGG LFD) appears to be a marker of the cardiac form of human Chagas' disease since increased anti-R13 antibody levels are correlated with severe cardiomyopathy but not with other clinical signs $(1,18)$. The putative involvement of ribosomal $\mathrm{P}$ proteins in the autoimmune process of Chagas' disease is supported by recent data showing a high degree of homology between the amino acid sequence of a peptide present on the second loop of the human $\beta 1$-adrenergic receptor and the carboxy-terminal part of the $T$. cruzi ribosomal P0 protein (TcP0). Antibodies from chagasic patients immunopurified on human $\beta 1$-adrenergic receptor peptides were shown to exert a positive chronotropic effect in vitro on cardiomyocytes from neonatal rats (11). This effect was blocked by both the specific $\beta 1$ antagonist bisoprolol and the peptide $\mathrm{P} 0 \beta$ derived from the $\mathrm{TcP} 0 \mathrm{C}$ terminus. It was the first time that an immune response elicited through a molecular mimicry mechanism reproduced a functional autoreactive clinical sign. Our present goal was to determine if anti-TcP $2 \beta$ antibodies induced by TcP $2 \beta$ immunization of mice are able to exert a chronotropic effect in vitro on cardiocytes through stimulation of the $\beta 1$-adrenergic receptor. This experimental approach could unambiguously demonstrate the role of the anti-TcP $2 \beta$ antibodies in a context which is not influenced by the complex variables of actual $T$. cruzi 
infection like immunosuppression and polyclonal activation. To assess this hypothesis, we immunized mice with two TcP2 $\beta$ fusion proteins (glutathione $S$-transferase [GST]-TcP2 $\beta$ and histidine [Hist]-TcP2 $\beta$ ) and two different adjuvants (Freund's and Alu-Gel-S adjuvants) and then characterized the humoral $\mathrm{TcP} 2 \beta$ responses in individual mice, including the definition of B-cell epitopes. Furthermore, the functional activity of the anti-TcP2 $\beta$ antibodies was assessed using spontaneously beating neonatal rat heart myocytes. Humoral anti-TcP2 $\beta$ responses were also analyzed in mice chronically infected with small doses of parasites.

\section{MATERIALS AND METHODS}

Construction and expression of ribosomal TcP2 $\beta$ fusion proteins. A cDNA of 340 bp coding for the acidic ribosomal P1-P2 protein of T. cruzi was originally recovered by PCR from a recombinant $\lambda$ gt11-TcP2 $\beta$ clone $(31,41)$ and inserted into the $B a m \mathrm{HI} / E c o$ RI and $B g l \mathrm{II} / E c o$ RI sites of the expression vectors pGEX.1 (Pharmacia) and pTcrHistB (Invitrogen), respectively. pGEX-TcP2 $\beta$ encoded a 36/37-kDa fusion protein corresponding to GST and the 12-kDa ribosomal P2 protein. pTcrHist-TcP2 $\beta$ encoded a $14.5-\mathrm{kDa}$ fusion protein corresponding to TcP2 $\beta$ with an N-terminal hexahistidine tag. Both constructions were used to transform Escherichia coli TOP $10 \mathrm{~F}^{\prime}$ competent cells (Invitrogen). Expression of the proteins was induced by adding $1 \mathrm{mM}$ isopropyl- $\beta$-D-thiogalactopyranoside (IPTG).

Purification of recombinant $\mathrm{TcP} 2 \beta$ proteins and proteolytic cleavage. Two liters of an induced culture (E. coli transformed by pGEX-TcP2 $\beta$ or pTcrHist$\mathrm{TcP} 2 \beta$ ) was pelleted, resuspended in $20 \mathrm{ml}$ of binding buffer (phosphate-buffered saline [PBS; $\mathrm{pH} 7.2$ ] $-1 \%$ Triton X-100 for GST-TcP2 $\beta$ or $20 \mathrm{mM}$ Na phosphate $[\mathrm{pH} 7.8]-500 \mathrm{mM} \mathrm{NaCl}-0.05 \%$ Nonidet phosphate for Hist-TcP2 $\beta$ ), and lysed by sonication in the presence of a protein inhibitor cocktail. After centrifugation at $10,000 \times g$ for $30 \mathrm{~min}$, the supernatants (GST-TcP2 $\beta$ and Hist-TcP2 $\beta$ crude extracts) were affinity purified. GST-TcP2 $\beta$ crude extract was loaded onto a glutathione agarose column (Sigma) equilibrated in PBS, and the GST-TcP2 $\beta$ fusion protein was eluted with $50 \mathrm{mM}$ Tris- $\mathrm{HCl}(\mathrm{pH} 8.0)$ containing $5 \mathrm{mM}$ reduced glutathione. Hist-TcP2 $\beta$ crude extract was loaded onto Talon metal affinity resin (Clontech, Palo Alto, Calif.) equilibrated in binding buffer and eluted in accordance with the manufacturer's instructions.

The purity of recombinant fusion proteins was assessed by sodium dodecyl sulfate- $-10 \%$ and $-12.5 \%$ polyacrylamide gel electrophoresis analysis. Protein content was determined by the Bradford method (Bio-Rad Protein Assay; BioRad, Richmond, Calif.). To confirm that the purified recombinant protein showed the predicted sequence deduced from the nucleotide sequence (accession no. P23623; National Center for Biotechnology Information BLAST search), the $\mathrm{N}$-terminal amino acid sequence was directly analyzed as previously described (23; http://www2.perkin-elmer.com).

T. cruzi infection or immunization of mice. $\mathrm{C} 3 \mathrm{H} / \mathrm{HeJ}$ mice, 8 to 10 weeks old, that were bred at the Pasteur Institute were used for infection or immunization. Mice were infected by intraperitoneal injection of $10^{6}$ epimastigotes (T. cruzi strain CL) from stationary-phase cultures. Mice were bled every week from day 14 to day 150 postinfection (p.i.). Sex- and age-matched uninfected mice were used as normal controls. Parasitemia was determined with blood from the tail vein by optical microscopy (3).

The following three immunization protocols were used: (i) injection of $100 \mu \mathrm{g}$ of GST-TcP2 $\beta$ emulsified in complete Freund's adjuvant (CFA) (Difco Laboratories, Detroit, Mich.), followed by two boosts with $100 \mu \mathrm{g}$ of the same protein emulsified in incomplete Freund's adjuvant (Sigma Chemical Co., St. Louis, Mo.); (ii) injection of $100 \mu \mathrm{g}$ of GST-TcP2 $\beta$ emulsified in Alu-Gel-S adjuvant (Boehringer Ingelheim, Heidelberg, Germany) and two boosts under the same conditions; and (iii) injection of $100 \mu \mathrm{g}$ of Hist-TcP2 $\beta$ emulsified in CFA and two boosts with $100 \mu \mathrm{g}$ of the same protein emulsified in incomplete Freund's adjuvant. All of the injections were performed intraperitoneally. Sera from preimmunized mice were used as controls.

Enzyme-linked immunosorbent assay (ELISA) and isotype detection. Microwell plates (Nunc Immunoplates; Nunc, Roskilde, Denmark) were coated overnight at $4^{\circ} \mathrm{C}$ with Hist-TcP2 $\beta$ at $0.5 \mu \mathrm{g} / \mathrm{ml}$ or with total $T$. cruzi extract (35) at 5 $\mu \mathrm{g} / \mathrm{ml}$ in $50 \mu \mathrm{l}$ of PBS. Plates were washed three times with washing buffer (PBS [pH 7.4] containing $0.1 \%$ Tween 20) and then incubated with blocking buffer (PBS plus $1 \%$ gelatin) for $2 \mathrm{~h}$ at room temperature. Sera from infected or immunized mice were diluted 1:100 in blocking buffer (or in serial dilutions for titration), added to duplicate series of wells, and incubated for $2 \mathrm{~h}$ at room temperature. After washing, $50 \mu \mathrm{l}$ of peroxidase-labeled goat anti-mouse immunoglobulin G ( $\operatorname{IgG}$ ) antibodies (Southern Biotechnology) diluted 1:3,000 was dispensed into each well and incubated for $1 \mathrm{~h}$ at room temperature. Color was developed by addition to each well of $50 \mu \mathrm{l}$ of 2,2'-azinobis(3-ethylbenzthiazolinesulfonic acid) (ABTS) peroxidase substrate solution (Kirkegaard \& Perry Laboratories Inc., Gaithersburg, Md.) and incubation in the dark at $37^{\circ} \mathrm{C}$ for 10 min. Optical density (OD) was measured at 405 and $650 \mathrm{~nm}$ with a double-length automated plate reader (Molecular Devices, Medi-Sciences).

For antibody isotyping, the peroxidase-conjugated second antibodies against
TABLE 1. Peptide sequences used for mapping of the B-cell epitope on ribosomal $\mathrm{P} 2$ protein

\begin{tabular}{l} 
Sequence $^{a}$ \\
\hline 1 Peptide
\end{tabular}

${ }^{a}$ The amino terminus is shown at the left.

mouse IgG1 (diluted 1:2,000), IgG2a (1:2,000), IgG2b (1:2,000), and IgG3 (1: $1,000)$ (Southern Biotechnology) were added after incubation with mouse sera (1:100) and then incubated for $1 \mathrm{~h}$ at room temperature. Plates were washed and revealed as described above. Controls were performed with sera from uninfected or preimmunized mice $(\mathrm{OD}, \leq 0.05)$. Samples were defined as positive when the mean OD value of duplicate samples +2 standard deviations (SD) was above the mean OD value of control mice. The significance of differences between groups was assessed by the analysis of variance module of the STATVIEW program.

Epitope mapping. A set of 25 biotinylated peptides (Table 1) was purchased from Chiron Technologies. These 12-mer peptides covered the full sequence of recombinant $\mathrm{TcP} 2 \beta$ and overlapped every four amino acids. Peptides were fixed at $0.16 \mu \mathrm{M}$ to 96 -well plates (Nunc Maxisorb plates) coated with streptavidin (Sigma), and after extensive washes, incubation was performed at a 1:1,000 dilution with hyperimmune serum and at 1:500 with sera from chronically infected mice in accordance with the manufacturer's instructions. Peroxidaselabeled goat anti-mouse IgG (Southern Biotechnology) was used as described above, and OD was read at $405 \mathrm{~nm}$.

Structure predictions. Predictions of protein secondary structure (PHD server, EMBL; http://www.embl-heidelberg.de/predictprotein) were deduced from the primary structure and after multiple-sequence alignment of the ТcP2 $\beta$ sequence with about 105 related ribosomal $\mathrm{P}$ sequences in the protein data bank (SwissProt) which offers the greatest accuracy.

Functional activity of purified antibodies. Cultured neonatal rat heart myocytes were used as a functional test system (42). Single cells were dissociated from the minced heart ventricles of 1- to 3-day-old Wistar rats with a $0.20 \%$ solution of trypsin. The myocytes were cultured as a monolayer on the bottom $\left(12.5 \mathrm{~cm}^{2}\right)$ of 25 -ml Falcon flasks $\left(1.6 \times 10^{6}\right.$ seeded cells in $2.5 \mathrm{ml}$ of medium $)$ with SM 20-I medium (Max Delbrück Center for Molecular Medicine, Berlin, Germany) containing $10 \%$ heat-inactivated calf serum and $2 \mu \mathrm{M}$ fluorodeoxyuridine (the latter prevented the proliferation of nonmuscle cells). They were cultured for 4 days at $37^{\circ} \mathrm{C}$ in air. The spontaneous beating frequency of myocytes was measured at $37^{\circ} \mathrm{C}$ on the heated stage of an inverted microscope. Changes in the beating frequency were measured $1 \mathrm{~h}$ after the addition of the purified IgG fractions prepared from mouse serum samples or 5 min after the addition of drugs. The basal beating rate was $162 \pm 24$ beats $/ \mathrm{min}$. IgG fractions were prepared from normal mouse sera or from sera of infected or hyperimmunized mice by precipitation with a saturated ammonium sulfate solution $(60 \%$ serum plus $40 \%$ ammonium sulfate). This precipitation was repeated thrice, the last pellet was solubilized in PBS, and the solution was dialyzed in the same buffer to remove small active peptides and hormones. The concentration of the purified IgG was adjusted to that of the original serum sample. In the experiment, the $\operatorname{IgG}$ fraction was used at a dilution 1:50. Individual sera were tested. 


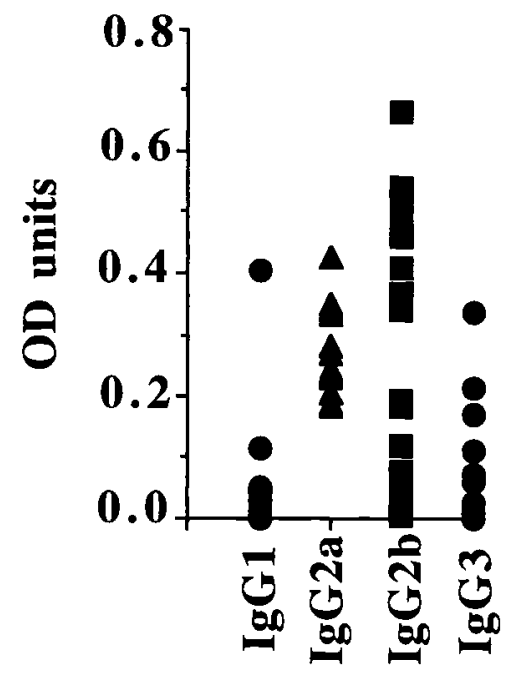

FIG. 1. Isotyping of sera $(n=15)$ from $T$. cruzi-infected mice. Sera diluted 100 times were tested individually, and the background values (mean value of normal controls $+2 \mathrm{SD}$ ) were subtracted for each datum point.

\section{RESULTS}

Anti-TcP2 $\beta$ humoral response in $T$. cruzi-infected mice. The humoral response against a total $T$. cruzi crude extract and against recombinant TcP $2 \beta$ of sera from $T$. cruzi-infected mice was maximal at 2 months p.i. (data not shown). Anti-TcP2 $\beta$ antibody titers, determined in the chronic phase of the experimental disease (day 134 p.i.), ranged from 1:500 to 1:6,000 (data not shown). As previous observations showed a predominance of $\mathrm{IgG} 2$ isotypes in $T$. cruzi-infected mice (35), we determined the anti-TcP $2 \beta$ antibody isotypes showing low levels of specific IgG1 and IgG3 and relatively high levels of IgG2b and IgG2a (Fig. 1).

Anti-TcP2 $\beta$ humoral response in GST-TcP2 $\beta$ - and HistTcP2 $\beta$-immunized mice. To investigate the humoral response against ribosomal TcP $2 \beta, \mathrm{C} 3 \mathrm{H} / \mathrm{HeJ}$ mice were immunized with GST-TcP $2 \beta$ in CFA or Alu-Gel-S or with Hist-TcP2 $\beta$ in CFA. The anti-TcP $2 \beta$ antibody level was assessed 15 days after the third boost on Hist-TcP $2 \beta$-coated ELISA plates. All immunized mice produced anti-TcP $2 \beta$ antibodies whose IgG titers ranged from 1:2,000 (GST-TcP2 $\beta$-CFA-immunized mice) to 1:50,000 (Hist-TcP2 $\beta$-CFA-immunized mice) (Fig. 2). Interestingly, we found that the type of adjuvant used in immunizations with the same protein influenced the antibody titers. Indeed, mice immunized with GST-TcP $2 \beta$ emulsified in CFA showed a lower antibody titer that those immunized with GST$\mathrm{TcP} 2 \beta$ in AluGel-S.

For the anti-TcP $2 \beta$ IgG isotypes of mice immunized with the three different protocols, see Fig. 3. Mice immunized with GST-TcP $2 \beta$ produced a high level of IgG1 and significant levels of IgG2a and IgG2b but not IgG3 independently of the adjuvant used for immunizations (Fig. $3 \mathrm{~A}$ and $\mathrm{B}$ ). In contrast, immunization with Hist-TcP2 $\beta$ elicited a significant level of IgG3 (Fig. 3C).

Epitope mapping. Peptide recognition was determined by testing sera from individual TcP $2 \beta$-immunized mouse collected 2 weeks after the last boost. Figure 4 shows the peptide recognition patterns of sera from $T$. cruzi-infected or recombinant TcP $2 \beta$-immunized mice. Two regions of the protein were reproducibly recognized by all of the TcP $2 \beta$-immunized mice tested. In particular, peptides 10 and 11 and peptides 16 and 17 (central region) were recognized with a high OD. This peptide profile was maintained for at least 9 months after the

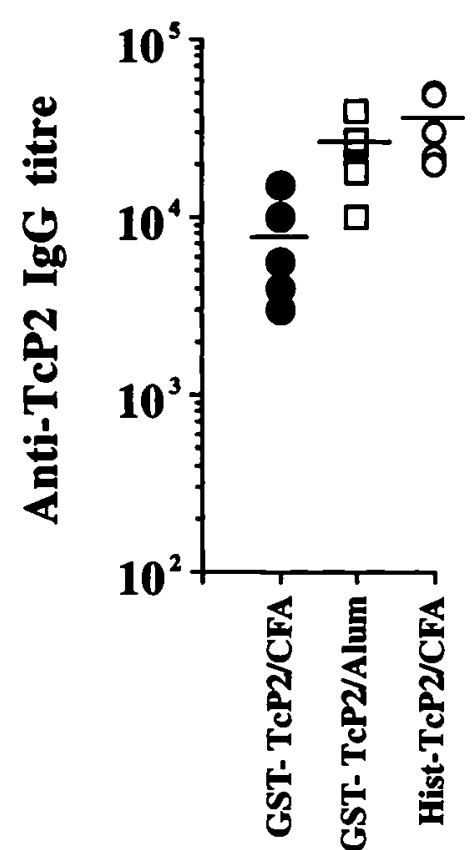

FIG. 2. Titration of sera from GST-TcP2 $\beta$-CFA (-)-immunized; GSTTcP $2 \beta-A l u-G e l-S-i m m u n i z e d ~(\square)$, and Hist-TcP2 $\beta$-CFA-immunized $(\bigcirc)$ mice. The mean value for each group is represented by a horizontal bar.

last boost, suggesting that these epitopes in the recombinant protein are strongly immunogenic. The TcP $2 \beta \mathrm{C}$ terminus (peptides 24 and 25 ) was occasionally recognized by some GST-TcP2 $\beta$-immunized mice with a lower OD ( 3 out of 10 mice analyzed in groups $\mathrm{A}$ and $\mathrm{B}$ ). In contrast, all Hist-TcP $2 \beta$ immunized mice (group $C$ ) were able to recognize this $\mathrm{C}$ terminal domain at a higher OD.

The peptide profiles recognized by sera from $T$. cruzi-infected mice were strikingly different (group D) from that of immunized mice (groups A, B, and C). Indeed, specific antibodies tested 134 days p.i. showed that sera from chronically infected mice recognized mainly the two C-terminal overlapping peptides which encompass the R-13 sequence $(1,18,24)$. This anti-C-terminal response arose late in infection, beginning at 60 days p.i., and was completely established in the chronic phase, remaining high throughout the life of the infected mice. Therefore, the different immunological environments in TcP2 $\beta$-immunized and $T$. cruzi-infected mice, where
A

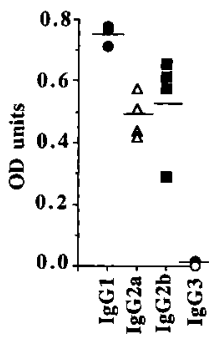

B

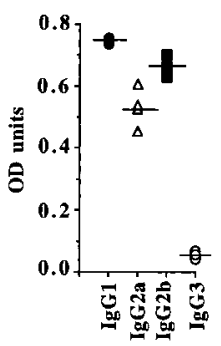

C

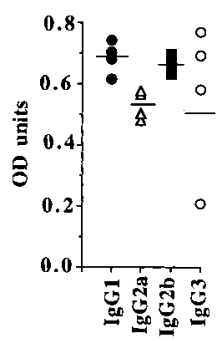

FIG. 3. Distribution of anti-TcP $2 \beta$ IgG isotypes from individual mice immunized with GST-TcP $2 \beta-$ Alu-Gel-S (A), GST-TcP $2 \beta-C F A(B)$, and Hist-TcP $2 \beta-$ CFA (C). Background values (OD units for maltose-binding protein and GST responses) have been subtracted for each datum point, and positive values correspond to those higher than the mean value of normal controls $+2 \mathrm{SD}$. 


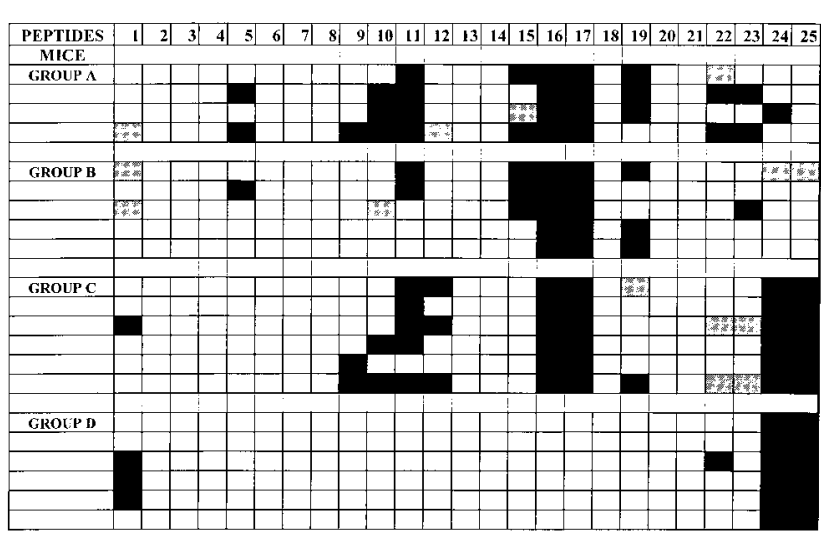

FIG. 4. Epitope mapping of sera from mice immunized with GST-TcP $2 \beta$ in CFA (group A) or Alu-Gel-S (group B) or with Hist-TcP $2 \beta$ in CFA (group C) and from chronically $T$. cruzi-infected mice (group D). Sera were tested at dilutions of 1:1,000 (immunized mice) and 1:500 (infected mice). Biotinylated 12 -mer peptides spanning the TcP2 $\beta$ molecule (no. 1 to 25 ) were used at $1 \mu \mathrm{g} / \mathrm{ml}$. Black, strong response; grey, weak response.

polyclonal activation persisted indefinitely, could account for the different B-cell responses observed in the two systems.

TcP2 $\beta$ molecule secondary-structure prediction. To test the hypothesis that antigenic residues are localized on external domains of the molecule which are preferentially exposed to the immune system, the predicted secondary structure of the TcP2 $\beta$ molecule was determined (Fig. 5). The structure belongs to $\alpha$ class aII, with four out of five $\alpha$ helices displaying a high reliability of prediction. The most likely topology for $\mathrm{TcP} 2 \beta$ would be the four-helix bundle. In addition, the prediction of buried and exposed residues (Fig. 5, P 3 ) suggested that most antigenic sites, covered by peptides 10 and 11 and peptides 16 and 17, were likely to be located on the surface of the bundle.

Functional activity test. Purified IgGs from sera of infected or immunized mice were assayed for functional recognition of the $\beta 1$ adrenoreceptor using spontaneously beating neonatal heart myocytes. This system has already been used for the study of antireceptor autoantibodies in idiopathic dilated cardiomyopathy (42), and the amino acid sequences of the second extracellular loop of the rat and human $\beta 1$-adrenergic receptors are identical (20). Therefore, it is a suitable model for studies of cross-reactive antibodies on heart cells. After $60 \mathrm{~min}$ of incubation with the purified IgG containing anti-TcP $2 \beta \mathrm{C}$ terminus antibodies, a significant increase in the beating frequency of heart myocytes was observed whereas no increase in the beating frequency was observed when antibodies were used that are not reactive with the TcP2 $\beta \mathrm{C}$ terminus (Table 2 ). It is

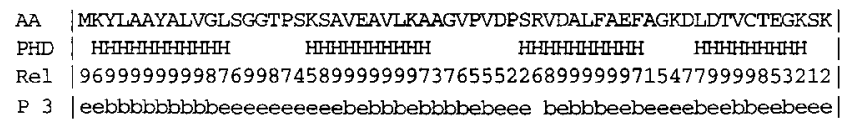

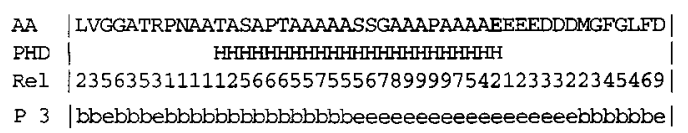

FIG. 5. Prediction of the secondary structure of TcP2 $\beta$ based on the 105 best local alignments. AA, amino acid sequence; PHD, profile network prediction sequence ( $\mathrm{H}$ denotes $\alpha$-helical positions); Rel, reliability index of prediction ranging from 0 to $9 ; \mathrm{P} 3$, relative solvent accessibility in three states (b, 0 to $9 \%$; e, 36 to $90 \%)$.
TABLE 2. Agonist-like effect of anti-TcP2 $\beta \mathrm{C}$ terminus antibodies ${ }^{a}$

\begin{tabular}{lccc}
\hline $\begin{array}{c}\text { Mouse } \\
\text { group }\end{array}$ & $\begin{array}{c}\text { Purified } \\
\text { antibodies }\end{array}$ & $\begin{array}{c}\text { Antibodies }+ \\
\text { bisoprolol }\end{array}$ & $\begin{array}{c}\text { Antibodies }+ \\
\text { atropine }\end{array}$ \\
\hline Control & $1.08 \pm 0.88$ & $\mathrm{ND}^{b}$ & $0.4 \pm 0.92$ \\
B1 & $2.2 \pm 1.16$ & $\mathrm{ND}$ & $2.0 \pm 1.24$ \\
$\mathrm{C} 1$ & $12.8 \pm 1.32$ & $0.6 \pm 1.16$ & $18 \pm 1.56$ \\
$\mathrm{C} 2$ & $16.6 \pm 1.36$ & $0.6 \pm 1.56$ & $21.2 \pm 1.4$ \\
D1 & $20.8 \pm 1.04$ & $12 \pm 1.12$ & $26.8 \pm 1.04$ \\
\hline
\end{tabular}

${ }^{a}$ Anti-TcP2 $\beta$ IgGs from sera were selected from GST-TcP2 $\beta$-Alu-Gel-S (group B)- or Hist-TcP2 $\beta$-CFA (group C)-immunized mice and chronically T. cruzi-infected mice (group D). The increase in beating frequency per minute was determined, and the mean \pm standard error of the mean of 10 different measurements is shown. Bisoprolol $(1 \mu \mathrm{M})$ and atropine $(1 \mu \mathrm{M})$ were added in different experiments.

${ }^{b} \mathrm{ND}$, not done.

noteworthy that the addition of the $\beta 1$-specific blocker bisoprolol was sufficient to completely block the positive chronotropic effect induced by anti-TcP $2 \beta \mathrm{C}$ terminus antibodies from Hist-TcP $2 \beta$-immunized mice, indicating that the agonistlike effect of the antibodies was realized by the recognition of the $\beta-1$ adrenoreceptor.

\section{DISCUSSION}

We have shown that sera from patients with chronic Chagas' heart disease displayed antibodies against the parasite ribosomal P1-P2 C terminus and that these antibodies are particularly abundant in patients with severe cardiomyopathy $(1,18)$. In addition, antibodies from patients with Chagas' disease have been shown to bind to myocardium target cells and to modulate their activity through recognition of cardiomyocyte surface receptors (i.e., $\beta$-adrenergic and muscarinic cholinergic receptors) $(2,10-13)$. It is assumed that molecular mimicry is responsible for this recognition of cardiac host molecules by parasite-elicited antibodies. In the present study, we investigated the B-cell response of mice immunized with two T. cruzi recombinant ribosomal $\mathrm{P} 2$ proteins and analyzed the B-cell epitopes and the functional activity of the antibodies related to specific epitopes. This response was compared with the anti$\mathrm{TcP} 2 \beta$ response in mice infected with the CL strain of T. cruzi. The specific anti-TcP $2 \beta$ humoral response of $T$. cruzi-infected mice presented in this report is similar to the human anti-R13 response based on B-cell epitope recognition $(1,18)$. The response was studied in humans by ELISA using the synthetic peptide R-13 (encompassing the 13 amino acids of the TcP2 $\beta$ $\mathrm{C}$ terminus) and in mice by ELISA using biotinylated overlapping peptides. In both studies, the unique epitope able to elicit antibodies is located at the $\mathrm{C}$ terminus. Regarding the B-cell epitopes of the anti-TcP $2 \beta$ response in mice immunized with different constructs of recombinant $\mathrm{TcP} 2 \beta$, we observed that they were quite different from those in infected mice. First, two groups of peptides located in the central part of the molecule are recognized following immunization with either GST$\mathrm{TcP} 2 \beta$ or Hist-TcP $2 \beta$, independently of the adjuvant used. These particular epitopes (peptides 10 and 11 and peptides 16 and 17) correspond to sequences outside of the four $\alpha$ helices of the molecule (Fig. 5). Secondly, mice immunized with GST$\mathrm{TcP} 2 \beta$ fail to recognize the $\mathrm{C}$ terminus of the protein. The mechanisms leading to these different responses are not yet understood, but the linkage to the protein carrier GST may interfere with epitope presentation by antigen-presenting cells. 
Besides, the absence of recognition of these internal epitopes in infected mice is possibly due to the immunosuppression occurring during the acute phase $(4,26)$ or to defective major histocompatibility complex class II presentation by $T$. cruzi-infected macrophages inducing a defective antigen-presenting cell function (17). Alternatively, the response against the conserved $\mathrm{C}$ terminus of $\mathrm{TcP} 2 \beta$ could parallel the intense B-cell polyclonal activation, increasing the level of autoantibodies during $T$. cruzi infection $(7,8)$. It is noteworthy that the IgG2a isotype was highly represented in the $\mathrm{TcP} 2 \beta$ response in immunized mice, as well as in infected mice, as previously described for nonspecific and parasite-specific humoral responses $(27,35)$. However, in immunized mice, the IgG1 involved in protection is usually the representative isotype $(5,9$, $30,36,37)$.

The interference by anti-TcP0 $\mathrm{C}$ terminus antibodies from chagasic patients with the functional activity of cardiocytes in vitro has been described by Ferrari et al. (11). Since these antibodies were immunopurified on a specific peptide belonging to the second external loop of the $\beta 1$-adrenergic receptor, it has been assumed that molecular mimicry was responsible for this interference. Moreover, this $\beta 1$-adrenergic receptor peptide has been described as a functional autoimmune epitope in idiopathic dilated cardiomyopathy $(21,22)$. Antibodies from mice chronically infected with $T$. cruzi demonstrated a chronotropic effect on rat neonatal cardiocytes partially due to $\beta 1$-adrenergic receptor recognition, as indicated by the decrease of activity in the presence of bisoprolol, a feature that was found preferentially in the acute phase of infection (25). The increase in functional activity in the presence of atropine argues for a muscarinic cholinergic activity present in infected mice, confirming these previous observations in mice (25) and humans (13). In mice immunized with recombinant TcP2 $\beta$, only antibodies from mice recognizing the $\mathrm{C}$ terminus are able to exert a chronotropic effect on spontaneously beating neonatal myocytes. This may be correlated with electrocardiogram modifications that occur after immunization with recombinant maltose-binding protein fusion $\mathrm{TcP} 2 \beta$ or $\mathrm{R}-13$ coupled to bovine albumin or egg albumin as previously described $(19,28)$ and with the presence of antiadrenoreceptor autoantibodies in chagasic cardiomyopathy. A monoclonal antibody specific for the TcP $2 \beta \mathrm{C}$ terminus is currently under investigation to determine the fine specificity of the epitope and its role after in vivo transfer.

\section{ACKNOWLEDGMENTS}

This work was supported by grant BIO4-CT96-5131 from the European Community. P. Sepulveda was a postdoctoral fellow supported by Marie Curie Research Training Grants (European Community Biotechnology Program) and the Plan de Formacion de Doctores en el Extranjero, Ministerio de Educacion y Cultura (Spain).

We thank P. Lopez-Bergami (Ingebi, Buenos Aires, Argentina) for providing the S-23 cDNA, J. D'Alayer (Institut Pasteur) for protein sequencing, and G. Levitus (Ingebi), M. Delarue, and S. Longacre (Institut Pasteur, Paris, France) for helpful advice.

\section{REFERENCES}

1. Aznar, C., P. Lopez-Bergami, S. Brandariz, C. Mariette, P. Liegeard, M. C. de Deus Alves, E. Luna Barreiro, R. Carrasco, S. Lafon, D. Kaplan, H. Miguez, C. Camacho, G. Levitus, M. J. Levin, and M. Hontebeyrie. 1995. Prevalence of anti-R-13 antibodies in human Trypanosoma cruzi infection. FEMS Immunol. Med. Microbiol. 12:231-238.

2. Borda, E., J. Pascual, P. Cossio, M. de la Vega, R. Arana, and L. SterinBorda. 1984. A circulating IgG in Chagas disease which binds to $\beta$-adrenoceptor of myocardium and modulates their activity. Clin. Exp. Immunol. 57:679-686.

3. Brener, Z. 1962. Therapeutic activity and criterion of cure on mice experimentally infected with Trypanosoma cruzi. Rev. Inst. Med. Trop. Sao Paulo 4:389-394.
4. Brener, Z., and A. U. Krettli. 1990. Immunology of Chagas' disease, p. 247-261. In D. J. Wiler (ed.), Modern parasite biology. W. H. Freeman \& Co., New York, N.Y.

5. Cerban, F., A. Gruppi, and E. Vottero-Cima. 1992. Trypanosoma cruzi: involvement of $\mathrm{IgG}$ isotypes in the parasitaemia control of mice immunized with parasite exoantigens of isoelectric points 4.5. Exp. Parasitol. 75:137-145.

6. Davies, J. M. 1997. Molecular mimicry: can epitope mimicry induce autoimmune disease? Immunol. Cell Biol. 75:113-126.

7. D'Imperio Lima, M. R., M. Hontebeyrie-Joskowicz, A. Coutinho, T. Kipnis, and H. Eisen. 1985. Very large isotypically atypical polyclonal plaque-forming cell responses in mice infected with Trypanosoma cruzi. Eur. J. Immunol. 15:201-203.

8. D'Imperio Lima, M. R., H. Eisen, P. Minoprio, M. Hontebeyrie-Joskowicz, and A. Coutinho. 1986. Persistence of polyclonal B-cell activation with undetectable parasitemia in late stages of experimental Chagas' disease. J. Immunol. 137:353-356.

9. El Bouhdidi, A., C. Truyens, M. T. Rivera, H. Bazin, and Y. Carlier. 1994. Trypanosoma cruzi infection in mice induces a polyisotypic hypergammaglobulinaemia and parasite-specific response involving high IgG2a concentrations and highly avid IgG1 antibodies. Parasite Immunol. 16:69-76.

10. Elies, R., I. Ferrari, G. Wallukat, D. Lebesgue, P. Chiale, M. Elizari, M. Rosenbaum, J. Hoebeke, and M. J. Levin. 1996. Structural and functional analysis of the B cell epitopes recognized by anti-receptor autoantibodies in patients with Chagas disease. J. Immunol. 157:4203-4211.

11. Ferrari, I, M. J. Levin, G. Wallukat, R. Elies, D. Lebesgue, P. Chiale, M Elzari, M. Rosenbaum, and J. Hoebeke. 1995. Molecular mimicry between the immunodominant ribosomal protein P0 of Trypanosoma cruzi and a functional epitope on the human $\beta 1$-adrenergic receptor. J. Exp. Med. 182: 59-65.

12. Goin, J. C., E. Borda, C. Perez Leiros, R. Storino, and L. Sterin-Borda. 1994. Identification of antibodies with muscarinic cholinergic activity in human Chagas disease: pathological implications. J. Auton. Nerv. Sys. 47:45-52.

13. Gorelik, G., A. M. Genaro, L. Sterin-Borda, S. G. Cappa, and E. S. Borda. 1990. Antibodies bind and activate $\beta$-adrenergic and cholinergic lymphocyte receptors in Chagas' disease. Clin. Immunol. Immunopathol. 55:221-236.

14. Gruber, A., and B. Zingales. 1993. Trypanosoma cruzi: characterization of two recombinant antigens with potential application in the diagnosis of Chagas' disease. Exp. Parasitol. 76:1-12.

15. Kerner, N., P. Liegeard, M. J. Levin, and M. Hontebeyrie-Joskowicz. 1991. Trypanosoma cruzi: antibodies to MAP-like protein in chronic Chagas' disease cross-react with mammalian cytoskeleton. Exp. Parasitol. 73:451-459.

16. Kierszenbaum, F. 1999. Chagas' disease and the autoimmunity hypothesis. Clin. Microbiol. Rev. 12:210-223.

17. La Flamme, A. C., S. J. Kahn, A. Y. Rudensky, and W. C. Van Voorhis. 1997. Trypanosoma cruzi-infected macrophages are defective in major histocompatibility complex class II antigen presentation. Eur. J. Immunol. 27:30853094.

18. Levitus, G., M. Hontebeyrie-Joskowicz, M. H. V. Van-Regenmortel, and M. J. Levin. 1991. Humoral autoimmune response to ribosomal P protein in chronic Chagas' disease. Clin. Exp. Immunol. 85:413-417.

19. Lopez-Bergami, P., P. Cabeza Meckert, D. Kaplan, G. Levitus, F. Elias, F. Quintana, M. Van Regermortel, R. Laguens, and M. J. Levin. 1997. Immunization with recombinant Trypanosoma cruzi ribosomal P2 $\beta$ protein induces changes in the electrocardiogram of immunized mice. FEMS Immunol. Med. Microbiol. 18:75-85.

20. Machida, C. A., J. R. Bunzow, R. P. Searles, H. V. Tol, B. Tester, K. A. Neve, P. Teal, V. Nipper, and O. Civelli. 1990. Molecular cloning and expression of the rat $\beta 1$-adrenergic gene. J. Biol. Chem. 265:12960-12965.

21. Magnusson, Y., S. Marullo, S. Höyer, F. Waagstein, B. Andersson, A. Vahlne, J. G. Guillet, A. D. Strosberg, A. Hjalmarson, and J. Hoebeke. 1990. Localization of a functional autoimmune epitope on the $\beta 1$-adrenergic receptor in patients with idiopathic dilated cardiomyopathy. J. Clin. Investig. 86:1658-1663.

22. Magnusson, Y., G. Wallukat, F. Waagstein, A. Hjalmarson, and J. Hoebeke. 1994. Autoimmunity in idiopathic dilated cardiomyopathy: characterization of antibodies against the $\beta 1$-adrenoceptor with positive chronotropic effect. Circulation 89:2760-2767.

23. Matsudaira, P. 1993. A practical guide to protein and peptide purification for microsequencing, 2nd ed. Academic Press, Inc., New York, N.Y.

24. Mesri, E. A., G. Levitus, M. Hontebeyrie-Joskowicz, M. H. V. Van Regenmortel, and M. J. Levin. 1990. Major Trypanosoma cruzi antigenic determinant in Chagas' heart disease shares homology with the systemic lupus erythematosus ribosomal P protein epitope. J. Clin. Microbiol. 28:1219-1225.

25. Mijares, A., L. Verdot, N. Peineau, B. Vray, J. Hoebeke, and J. Argibay. 1996. Antibodies from Trypanosoma cruzi infected mice recognize the second extracellular loop of the $\beta 1$-adrenergic and M2-muscarinic receptors and regulate calcium channels in isolated cardiomyocytes. Mol. Cell. Biochem. 163/164:107-112

26. Minoprio, P., S. Itohara, C. Heusser, S. Tonegawa, and A. Coutinho. 1989. Immunobiology of murine Trypanosoma cruzi infection: the predominance of parasite-nonspecific responses and the activation of TCR1 T cells. Immunol. Rev. 112:183-207. 
27. Minoprio, P. M., O. Burlen, P. Pereira, B. Guilbert, L. Andrade, M. Hontebeyrie-Joskowicz, and A. Coutinho. 1988. Most B cells in acute Trypanosoma cruzi infection lack parasite specificity. Scand. J. Immunol. 28:553-561.

28. Motran, C. C., F. M. Cerban, W. Rivarola, D. Iosal, and E. V. de Cima. 1998. Trypanosoma cruzi: immune responses and functional heart damage induced in mice by the main linear B-cell epitope of parasite ribosomal P proteins. Exp. Parasitol. 88:223-230.

29. Oldstone, M. B. A. 1987. Molecular mimicry and autoimmune disease. Cell 50:819-820.

30. Pyrrho, A. S., J. L. C. Moraes, L. M. T. Peçanha, and C. R. Gattass. 1998. Trypanosoma cruzi: IgG1 and IgG2b are the main immunoglobulins produced by vaccinated mice. Parasitol. Res. 84:333-337.

31. Schijman, A. G., M. P. Vazquez, C. Ben Dov, S. Ghio, H. Lorenzi, and M. J. Levin. 1995. Cloning and sequence analysis of the TcP2 $\beta$ variants of Trypanosoma cruzi. Biochim. Biophys. Acta 1264:15-18.

32. Schmunis, G. A. 1987. Autoimmunity in Chagas' disease. Mem. Inst. Oswaldo Cruz 82(Suppl. 1):287-310.

33. Skeiky, Y. A. W., D. R. Benson, J. A. Guderian, P. R. Sleath, M. Parsons, and S. G. Reed. 1993. Trypanosoma cruzi acidic ribosomal P protein gene family. J. Immunol. 151:5504-5515.

34. Smith, H. R., and A. D. Steinberg. 1986. Autoimmunity-a perspective. Annu. Rev. Immunol. 1:175-210.

35. Spinella, S., P. Liegeard, and M. Hontebeyrie-Joskowicz. 1992. Trypanosoma cruzi: predominance of IgG2a in nonspecific humoral response during experimental Chagas' disease. Exp. Parasitol. 74:46-56.
36. Taibi, A., A. G. Espinoza, and A. Ouaissi. 1995. Trypanosoma cruzi: analysis of cellular and humoral response against a protective recombinant antigen during experimental Chagas' disease. Immunol. Lett. 48:193-200.

37. Takehara, H. A., A. Perini, M. H. Silva, and I. Mota. 1981. Trypanosoma cruzi: role of different antibody classes in protection against infection in the mouse. Exp. Parasitol. 52:137-146.

38. Tibbetts, R. S., T. S. McCormick, E. C. Rowland, S. D. Miller, and D. M. Engman. 1994. Cardiac antigen-specific autoantibody production is associated with cardiomyopathy in Trypanosoma cruzi-infected mice. J. Immunol. 152:1493-1499.

39. Van Voorhis, W. C., and H. Eisen. 1989. FL-160: a surface antigen of Trypanosoma cruzi that mimics mammalian nervous tissue. J. Exp. Med. 169: 641-648.

40. Van Voorhis, W. C., L. Barrett, R. Koelling, and A. G. Farr. 1993. FL-160 proteins of Trypanosoma cruzi are expressed from a multigene family and contain two distinct epitopes that mimic nervous tissues. J. Exp. Med. 178: 681-694.

41. Vazquez, M. P., A. G. Schijman, A. Panebra, and M. J. Levin. 1992. Nucleotide sequence of a cDNA encoding another Trypanosoma cruzi acidic ribosomal P2 type protein (TcP2 $\beta$ ). Nucleic Acids Res. 20:2893.

42. Wallukat, G., and A. Wollenberger. 1987. Effects of the serum gammaglobulin fraction of patients with allergic asthma and dilated cardiomyopathy on chronotropic $\beta$-adrenoreceptor function in cultured neonatal rat heart myocytes. Biomed. Biochim. Acta 46:634-639.

Editor: J. M. Mansfield 